\title{
Optical properties of GaSe semiconducting crystals intercalated by CdTe nanoparticles
}

\author{
Y.B. Khalavka ${ }^{1}$, L.P. Shcherbak ${ }^{1}$, M.M. Pyrlya ${ }^{2}$, V.B. Boledzyuk ${ }^{2}$ and Z.D. Kovalyuk ${ }^{2}$ \\ ${ }^{\prime} Y u$. Fed'kovych Chernivtsi National University, 2, Kotsyubinskyi str., 58012 Chernivtsi, Ukraine \\ ${ }^{2}$ I. Frantsevich Institute of Materials Science, NAS of Ukraine, Chernivtsi Branch, \\ 5, Iryna Vilde str., 58001 Chernivtsi, Ukraine
}

\begin{abstract}
The transmission spectra of GaSe crystals intercalated by CdTe nanoparticles (NPs) from aqueous colloidal solutions were investigated at $77 \mathrm{~K}$ in the range of GaSe excitonic absorption. Solutions of two types - yellow (with 1-2.5 nm NPs) and orange (with 2.5-3.5 nm NPs) - were used for the experiments. Essential GaSe optical density intensity growth in proportion to exposition time was observed after intercalation from yellow solutions up to 50 days. While the exciton peak position (near $2.1 \mathrm{eV}$ ) coincides both in "pure" GaSe and GaSe $\langle\mathrm{CdTe}\rangle$ after intercalation from yellow solution, the shortwave shift is observed for GaSe $\langle\mathrm{CdTe}\rangle$ intercalated from the orange solutions. The results are discussed in assumption of strain effect presence.
\end{abstract}

Keywords: semiconductors, nanoparticle, intercalation, light absorption, cadmium telluride, gallium monoselenide.

Manuscript received 01.04.09; accepted for publication 14.05.09; published online 15.05.09.

\section{Introduction}

Wide-bandgap cadmium telluride nanoparticles (NPs) synthesized by wet chemical routes are very promising objects for various applications including opto- and nanoelectronic devices [1]. However, little information is published about heterostructures prepared with "wet CdTe NPs".

Intercalated by various species, both by electrolytes and nonelectrolytes, crystals of layered semiconductor gallium monoselenide are appropriate objects for fundamental studies and are prospective for solving same applied problems (electrodes for fuel cells, hydrogen accumulators and others [2-4]). The layers in GaSe consist of four Ga-Ga-Se sheets with three $\mathrm{Se}$ atoms coordinated by $\mathrm{Ga}$ atom. The covalent $\mathrm{Ga}-\mathrm{Ga}$ bonds are oriented perpendicularly to the layers. In most cases, electrochemically introduced "guests" are situated in interlayered space overcoming weak Van-der-Waalse bonds [2-6] though some impurities can interact with the compound components, forming new compounds [7]. Availability of substances of new type, namely, nanoparticles, gives a possibility to enlarge the intercalant list and form heterostructures.

The aim of this work is to study the possibility of electrically charged [8] CdTe NPs electrochemical intercalation into the layered GaSe. In this paper, we present the investigations of absorption spectra of intercalated by the CdTe nanoparticles GaSe crystals measured in the range of $\mathrm{GaSe}$ excitonic absorption.

\section{Experimental}

Two series of aqueous colloidal CdTe solutions with the NPs size 1 to $2.5 \mathrm{~nm}$, a yellow solution (series 1) and 2.5-3.5 nm, an orange solution (series 2) were used for intercalation of the GaSe specimens. The CdTe NPs were prepared at room temperature by $\mathrm{H}_{2} \mathrm{Te}$-gas barboting into Ar-saturated $\mathrm{CdCl}_{2}$ solution containing $\mathrm{NaOH}$ to ensure $\mathrm{pH}=10-12$. Thioglicolic acid (TGA) was used as stabilizing agent in all the experiments. Post-preparative high-temperature $\left(100^{\circ} \mathrm{C}\right)$ treatment accelerated growth of nanoparticles and provided narrower particles size distribution. The CdTe nanoparticles sizes were estimated from optical absorption spectra.

The study of the CdTe NPs intercalation was carried out using GaSe single crystals grown by the modified Bridgman method from high pure components $(6 \mathrm{~N})$. The CdTe NPs intercalation into GaSe was performed electrochemically by means of the "drawing" electrical field method [2] in galvanostatic conditions. The concentration of the inserted impurity has been controlled by the value of electricity that was conducted 
through the electrochemical cell, i.e. electrical current density and duration of the intercalation were its checking parameters. The influence of the amount of inserted intercalant on the optical properties of gallium monoselenide was studied using a low current density (below $10 \mu \mathrm{A} / \mathrm{cm}^{2}$ ). The same samples were used for further intercalation by direct exposure of CdTe NPs from the yellow colloidal solution up to 50 days (series 1). Intercalation of the CdTe NPs from the orange solutions (series 2) was realized for 15 days.

Transmission spectra measurements have been performed using an IKS-31 spectrometer at $77 \mathrm{~K}$ and samples of 10 to $20 \mu \mathrm{m}$ thickness. The spectral resolution was better than $0.5 \mathrm{meV}$ for the investigated spectral range $2.02-2.18 \mathrm{eV}$. The light beam was directed along the $C$ axis of the samples.

\section{Results and discussion}

Influence of the intercalated into $\mathrm{GaSe}\langle\mathrm{CdTe}\rangle \mathrm{NPs}$, prepared in the series 1 , on the GaSe optical density is illustrated in Fig. 1. According to the data, growth of the GaSe optical density is proportional to the exposition time, i.e. to an inserted impurity concentration. Besides, the first exciton peak corresponding to the exciton state $n=1$ near the direct gap $E_{1}=(2.0954 \pm 0.0002) \mathrm{eV}$, and the second exciton peak $(n=2)$ near $E_{2}=2.12 \mathrm{eV}$ became more obvious with prolongation of the exposition time. It must be noted that the first exciton peak position (near $2.1 \mathrm{eV}$ ) coincides both in "pure" $\mathrm{GaSe}$ and $\mathrm{GaSe}\langle\mathrm{CdTe}\rangle$. The obtained results led to the conclusion about migration of some species into GaSe from the initial colloidal solution resulting in deformation processes or possibility of their interaction with the GaSe matrix components.

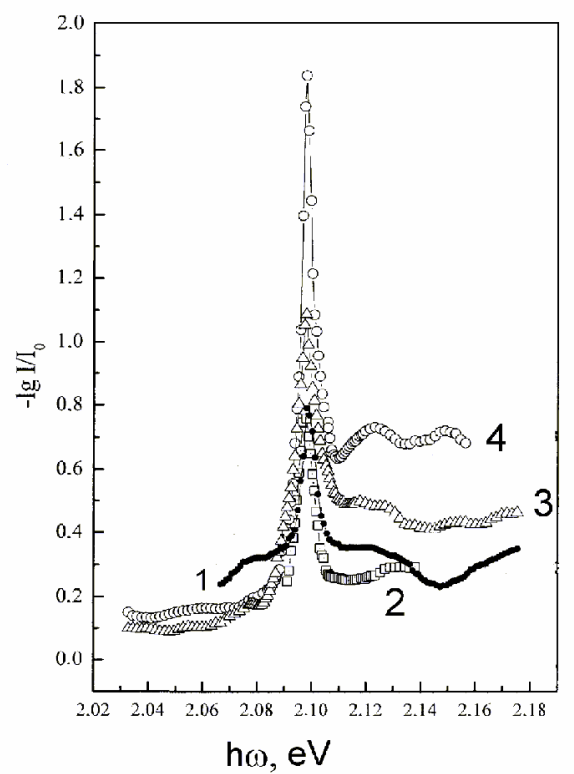

Fig. 1. Absorption spectra of "pure" GaSe (1) and GaSe intercalated from yellow solution for 7 (2), 21 (3) and 50 (4) days, which were measured at $77 \mathrm{~K}$.

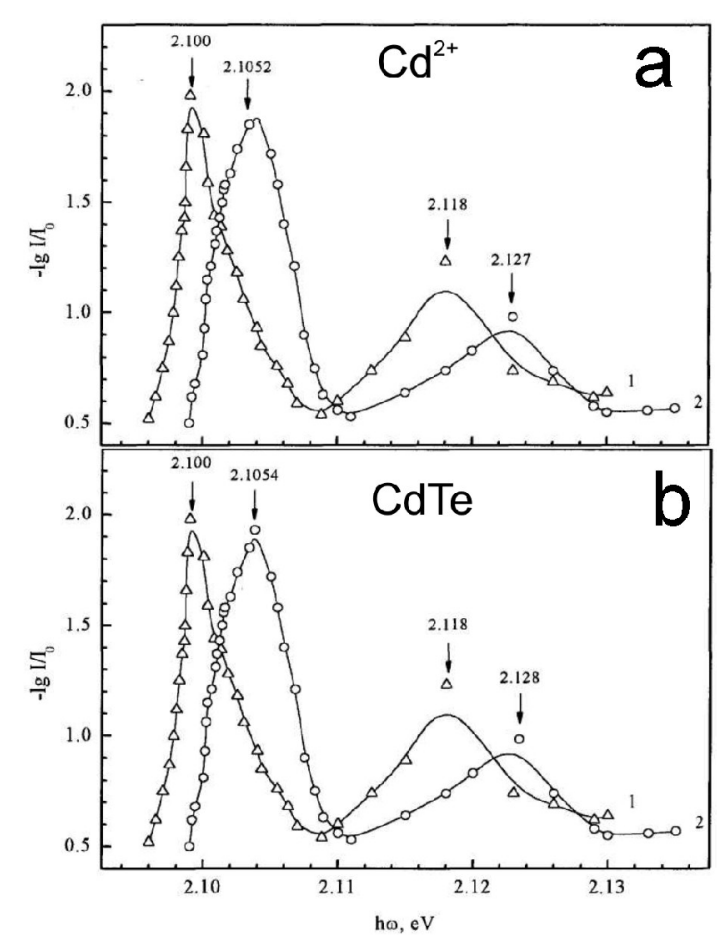

Fig. 2. Absorption spectra of "pure" $\mathrm{GaSe}(\Delta)$ as well as (a) GaSe intercalated from the $\mathrm{Cd}^{2+}$ solution (o) and (b) from the orange solution of CdTe nanoparticles (o).

Taking into account that GaSe and $\mathrm{GaSe}\langle\mathrm{CdTe}\rangle$ reflection coefficient $R(h v)$ is constant, the growth in the optical density can be related to an integral absorption coefficient $S_{0}$. According to the data, $S_{0}$ changes can be attributed either to exciton-phonon interaction processes, low-energy optical phonon existence or direct and indirect vertical transitions [9].

To determine influence of other components of the colloidal solutions, partly, cations $\mathrm{Cd}^{2+}, \mathrm{Na}^{+}$, anions $\mathrm{Cl}^{-}$, $\mathrm{OH}^{-}$and thioglicolic acid as possible intercalants, second series of experiment was performed with the orange solution (series 2) containing larger CdTe NPs. Various combinations of the intercalation solution content were used in these investigations.

Comparison of the absorption spectra of the initial GaSe sample and the same ones after intercalation from the orange solution (Fig. 2) show shortwave shift both of $E_{1}$ and $E_{2}$ by $\Delta E_{\mathrm{exc}}^{n=1}=4.6 \mathrm{meV}$ and $\Delta E_{\mathrm{exc}}^{n=2}=6.4 \mathrm{meV}$, accordingly. Contrary, electrochemical intercalation with anode polarization resulted only in $\Delta E_{\mathrm{exc}}^{n=1}=4.1 \mathrm{meV}$ (see Table).

Supposing that the interlayer distance is smaller than the CdTe NPs sizes from the orange solutions, electrochemical intercalation was performed with the same content of the precursors but without CdTe NPs. Shortwave shift parameters changed to $\Delta E_{\text {exc }}^{n=1}=$ $4.3 \mathrm{meV}$ and $\Delta E_{\mathrm{exc}}^{n=2}=7 \mathrm{meV}$, accordingly, in this case. However, GaSe samples kept for 15 days in the orange 
Table. Parameters of GaSe exciton energy shift as a function of the intercalation process conditions.

\begin{tabular}{|c|c|c|c|}
\hline System & $\begin{array}{l}\text { Conditions of } \\
\text { intercalation }\end{array}$ & $\begin{array}{c}\Delta E_{\mathrm{exc}}^{\mathrm{n}=1} \\
\mathrm{meV}\end{array}$ & $\begin{array}{c}\Delta E_{\mathrm{exc}}^{\mathrm{n}=2} \\
\mathrm{meV}\end{array}$ \\
\hline CdTe-TGA-Cd $^{2+}$ & $\begin{array}{c}\text { Cathode } \\
\text { polarization }\end{array}$ & 4.6 & 6.4 \\
\hline CdTe-TGA-Cd $^{2+}$ & Anode polarization & 4.1 & \\
\hline CdTe-TGA-Cd $^{2+}$ & Auto intercalation & 5.4 & 10 \\
\hline $\mathrm{Cd}^{2+}$, TGA & Auto intercalation & 5.2 & 9 \\
\hline $\mathrm{Cd}^{2+}, \mathrm{TGA}$ & $\begin{array}{c}\text { Cathode } \\
\text { polarization }\end{array}$ & 4.3 & 7 \\
\hline $\mathrm{Cd}^{2+}$ & $\begin{array}{c}\text { CdTe NPs } \\
\text { synthesis in situ }\end{array}$ & 3.2 & \\
\hline $\mathrm{Cd}^{2+}$ & 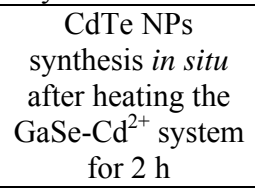 & 4.2 & \\
\hline
\end{tabular}

solution and identical solution but without CdTe NPs (auto intercalation) revealed even more pronounced shortwave shift, especially for $\Delta E_{\text {exc }}^{n=2}$ (see Table). Probably, this fact witnesses to some interlayered deformations in the used solutions.

Two experiments were carried out to form CdTe NPs in situ, saturating GaSe samples by $\mathrm{H}_{2}$ Te-gas after their holding in a $\mathrm{Cd}^{2+}$ aqueous solution only (without $\mathrm{NaOH}$, TGA, CdTe NPs). One such experiment differs from another by the previous $\left(\mathrm{GaSe}+\mathrm{Cd}_{\mathrm{aq}}^{2+}\right)$ sample that was heated at $100^{\circ} \mathrm{C}$ for the $\mathrm{Cd}^{2+}$ content enlargement in the layered compound. The optical spectra of the $\left(\mathrm{GaSe}+\mathrm{Cd}_{\mathrm{aq}}^{2+}\right)$ sample did not reveal any evident changes, while after its saturation by $\mathrm{H}_{2} \mathrm{Te}$ the single shift $\Delta E_{\mathrm{exc}}^{n=1}=3.2$ or $4.2 \mathrm{meV}$, accordingly, was observed (see Table).

To approve some conclusions about nature of the inserted species, one need direct diffraction investigations of the obtained objects. However, taking into account the peculiarities of the GaSe crystalline structure, one can point out that contribution of various type deformations (within separate layers and between them) to modification of the GaSe band structure. The excitonic peak shift to higher energies by $7 \mathrm{meV}$ in some experiments can take place due to interlayer changes of the elastic constants that lead to an increase of the energy gap $E_{g}$. Probably, it can be explained by effective mass change during intercalation. In a general case, $E_{g}$ variation is a result of competitive contributions of interand intralayer deformations. The shift of $E_{\text {exc }}$ can be induced by several factors, namely: 1) change of the interlayer elastic constants; 2) variation of the exciton binding energy during intercalation, preferably due to effective mass changes; 3) band-to-band transitions with a participation of the density-of-states tail; 4) fluctuations of the energy gap edges affected by "random" field of the inserted impurity.

\section{References}

1. M. Gao, C. Lesser, S. Kirstein, A.L. Rogach, and H. Weller // J. Appl. Phys. 87, p. 2302 (2000).

2. I.D. Kozmik, Z.D. Kovalyuk, I.I. Grigorchak, and B.P. Bachmatyuk // Neorganich. materialy 23, p. 754 (1987) (in Russian).

3. Z.D. Kovalyuk, T.P. Prokipchuk, A.I. Seredyuk, and K.D. Tovstyuk // Fizika Tverd. Tela 29, p. 2191 (1987) (in Russian).

4. Z.D. Kovalyuk, M.M. Pyrlya, and V.B. Boledzyuk // J. Optoelectron. Adv. Mater. 5, p. 869 (2003).

5. V.K. Lukyanyuk, S.P. Voronyuk, and Z.D. Kovalyuk // Phys. status solidi (b) 155, p. 717 (1989).

6. I.I. Grigorchak, V.V. Netyaga, and Z.D. Kovalyuk // J. Phys.: Condens. Matter 9, L191 (1997).

7. Yu.I. Zhirko, I.P. Zharkov, M.M. Pyrlya, V.B. Boledzyuk and Z.D. Kovalyuk // Semiconductor Physics, Quantum Electronics and Optoelectronics 7(4), p. 404 (2004).

8. A.L. Rogach, N.A. Kotov, D.S. Koktysh, J.W. Ostrander, and G.A. Ragoisha // Chem. Matter. 12, p. 2721 (2000).

9. F. Levi (Ed.), Intercalated Layered Materials. D. Reidel Publ. Co., Dordrecht, 1979. 